\title{
Comportements Reproductifs Des Jeunes Femmes A Bafia Au Cameroun
}

\author{
Didier Nganawara \\ H. Blaise Nguendo Yongsi
}

Institut de Formation et de Recherche Démographiques (IFORD),

Yaoundé, Cameroun

doi: 10.19044/esj.2017.v13n18p386 URL:http://dx.doi.org/10.19044/esj.2017.v13n18p386

\begin{abstract}
Background: With regard to sexual and reproductive health policies, the young are usually a target population. In fact, reproductive behaviours of young people often constitute a matter of public health concern, as their well-being is hampered by early and unwanted pregnancies, unsafe abortions along with complications, HIV/AIDS. Objective: This study aims at providing a better insight into reproductive behaviours of two groups of women (15-24 years and 25-34 years) in Bafia, and to link them with their cultural, economic, and socio-demographics characteristics. Results: The analyses performed show that women aged 15-24 years enter prematurely the sexual and fertile life more than those aged 25-34 years. For those who are still singles, the mean age at first pregnancy is 17,7 years for women aged 15-24 years versus 20,1years for those aged 25-34 years. And as for married women, the mean age at first pregnancy is respectively of 17 ,6years versus 19,4 years. However, women aged 15-24 years get married more lately than those aged 25-34 years. Conclusion: Although the level of knowledge of the contraceptive methods of women aged 25-34 years is too high than those aged 15-24 years, it appears that the older age-class (25-34 years) resort less to contraceptive methods than the younger old-class (15-24 years), whatever their matrimonial status. However, the standard of life and level of education act in an indisputable on those indicators.
\end{abstract}

Keyswords: Reproductive behaviours, young women, biographical approach, Bafia

\section{Resume}

Contexte: Les jeunes constituent un groupe cible privilégié des politiques en matière de santé sexuelle et reproductive. En effet, leurs comportements reproductifs posent souvent un problème de santé publique parmi lesquels, les grossesses précoces ou non désirées et ses complications, 
les avortements provoqués, l'exposition au VIH/Sida. Objectif : cette étude cherche à mieux cerner les comportements reproductifs de deux groupes de générations de femmes (15-24 ans et 25-34 ans) à Bafia, et de les mettre en relation avec leurs caractéristiques sociodémographiques, culturelles et économiques. Méthode: Les données utilisées proviennent de l'enquête biographique réalisée en 2010 sur la pauvreté et les besoins non satisfaits en santé reproductive des adolescents et jeunes. Résultats : Les différentes analysent montrent que les femmes de 15-24 ans entrent précocement dans la vie sexuelle et féconde que celles de 25-34 ans. Pour les célibataires, l'âge moyen à la première grossesse est de 17,7 ans pour les 15-24 ans contre 20,1 ans pour les 25-34 ans, et pour les mariées, il est respectivement de 17,6 ans contre 19,4 ans. En revanche, les femmes de 15-24 ans entrent tardivement en union que celles de 25-34 ans. Conclusion: Bien que le niveau de connaissance des méthodes contraceptives de ces dernières soit trop élevé que celui de leurs homologues de 15-24 ans, il semble que les plus âgées utilisent un peu moins ces méthodes que les plus jeunes, quel que soit leur statut matrimonial. Le niveau de vie et l'instruction agissent de manière incontestable sur ces indicateurs.

Mots clés : Comportements reproductifs, jeunes femmes, approche biographique, Bafia

\section{Introduction}

Le Cameroun, comme la plupart de nombreux pays, a ratifié le programme d'action de la Conférence Internationale sur la Population et le Développement (CIPD) de 1994. Lequel programme indique que les questions relatives à la santé sexuelle et reproductive des jeunes, ainsi que celles relatives aux droits liés à la reproduction et à l'autonomisation des femmes soient importantes pour un développement équilibré (Guillaume, 2004 ; Vimard, 2007). Ainsi, des années plus tard, en souscrivant aux Objectifs de Développement Durable (ODD), le Cameroun renouvelle son engagement à répondre aux besoins des adolescents et des jeunes en matière de la santé sexuelle et reproductive.

En dépit des politiques et programmes d'intervention sur les risques associés à l'activité sexuelle et reproductive des adolescents et des jeunes, la plupart des indicateurs montrent que les objectifs escomptés sont loin d'être atteints. En effet, le taux de prévalence contraceptive reste faible parmi les femmes en union, soit $23,4 \%$. L'âge d'entrée en union des femmes au Cameroun est assez précoce. Ainsi, en 2011, 17\% des femmes de 25-49 ans étaient déjà en union avant d'atteindre l'âge de 15 ans (BUCREP, 2011). La proportion des femmes de 20-24 ans qui entrent en union avant l'âge de 18 ans est de $38 \%$. En outre, avant d'atteindre l'âge de 15 ans, $21 \%$ des femmes 
de 25-49 ans avaient déjà eu des rapports sexuels et cette proportion est de $81 \%$ avant l'âge de 20 ans. L'âge médian aux premiers rapports sexuels chez les femmes de 25-49 ans est estimé à 17,0 ans. Il est de 17,3 ans pour les femmes âgées de 20-24 ans. L'intensité de l'activité sexuelle augmente le risque de grossesse et, partant, la fécondité des adolescentes. Ces dernières constituent un groupe à risque en matière de fécondité : en effet, la fécondité précoce a souvent des effets négatifs sur la santé des enfants et des mères et la probabilité des enfants de décéder est d'autant plus grande qu'ils naissent des mères très jeunes (OMS, 1997: Beninguissé, 2007). Les statistiques indiquent que la fécondité précoce reste élevée avec un taux de 127 pour mille à 15-19 ans. Parmi les 20-24 ans, 5\% de femmes déclarent avoir déjà eu un avortement provoqué. Tous ces indicateurs s'inscrivent dans un contexte de pauvreté grandissante au Cameroun en général et à Bafia en particulier. Dans cette ville, la pauvreté affecte plus généralement les femmes qui souffrent plus de problèmes nutritionnels (Wang, 1998), d'une lourde charge de travail, d'un accès limité aux soins de santé, d'un manque d'éducation, de même que d'un manque d'accès aux opportunités économiques et sociales (INS, 2007 ; Bessala, 2015).

Cependant, plusieurs facteurs peuvent intervenir, à différents niveaux, pour expliquer les comportements procréateurs des jeunes femmes. Ceux-ci intègrent aussi bien le contexte général dans lequel évolue la jeune femme (tradition, religion, législation, degré de modernité, etc.), les caractéristiques des parents et des ménages (catégorie socioprofessionnelle, le niveau de vie, etc.) et les caractéristiques propres à la jeune femme (âge au premier rapport sexuel, âge à la première union, instruction, etc.). Pour Davis et Blake (1956), initiateurs du concept de variables intermédiaires de la fécondité au milieu des années 1950, toutes les autres variables d'ordre socioéconomique, environnemental et culturel susceptibles d'avoir une relation avec la fécondité passent nécessairement par le biais d'un ou de plusieurs déterminants immédiats qui, à leur tour influencent la fécondité. Ainsi, ces auteurs ont construit un cadre d'analyse de la fécondité dans lequel ces variables intermédiaires sont classées en trois rubriques selon qu'elles soient liées aux risques d'exposition, aux relations sexuelles, à la grossesse, à la gestation et à la parturition. Parmi ces variables, on note l'âge d'entrée en union, dont l'influence sur la fécondité n'est plus à démontrer en démographie (Schoumaker et Tabutin, 1999), le calendrier de l'activité sexuelle, et l'utilisation des méthodes contraceptives. En se référant à ce cadre d'analyse, cette étude cherche à faire une analyse différentielle des comportements reproductifs de deux groupes de générations de femmes (1524 ans et 25-34 ans) à travers l'âge d'entrée dans la vie sexuelle, l'âge au premier mariage, la connaissance et l'utilisation des méthodes contraceptives, 
l'âge à la première grossesse et à l'accouchement. De manière spécifique, il s'agit de :

Décrire l'entrée dans la vie sexuelle et en union des femmes âgées de 15-24 ans et 25-34 ans en fonction de leurs caractéristiques sociodémographiques, culturelles et économiques; Examiner les aspects différentiels des taux d'utilisation des méthodes contraceptives de ces deux groupes;

Décrire l'entrée dans la vie génésique de ces deux groupes de générations de femmes en relation avec leurs caractéristiques.

Cette étude s'articule autour de deux grandes sections. La première présente les données et la méthodologie adoptées pour atteindre nos objectifs, et la seconde est consacrée aux résultats de nos analyses ainsi que les commentaires y relatifs.

\section{Donnees et methodologie}

Les données proviennent de l'enquête biographique intitulée « Pauvreté et besoins non satisfaits en santé de la reproduction chez les adolescents et les jeunes résidant dans la ville de Bafia en 2010. Elles se rapportent au module sur l'histoire génésique et pratique contraceptive des femmes âgées de 15 à 34 ans ayant eu au moins une grossesse au cours de leur vie. Ce module a pour but de saisir les différentes grossesses de l'enquêtée, leur prise en charge et leur issue. Une série de questions sur les consultations prénatales, les signes de complications de grossesse, le personnel consulté, le lieu des soins prénatals, etc. permettait d'apprécier la qualité de la prise en charge. Des questions sur le désir d'espacer ou de limiter les naissances croisées avec la connaissance et l'utilisation des moyens contraceptifs permettaient d'appréhender la régulation de la fécondité. En outre, l'issue de chaque grossesse est aussi identifiée (naissance vivante, mort-né, fausse couche, avortement provoqué ou grossesse en cours). Pour les accouchements, on demandait le type de personnel ayant assisté à l'accouchement, le lieu de l'accouchement et si l'enquêtée avait suivi des visites postnatales.

Ces biographies sont collectées depuis l'âge de dix ans jusqu'au moment de l'enquête en 2010. Cette dernière a couvert 21 Zones de Dénombrement (ZD), 5340 ménages et 1579 jeunes âgés de 15 à 34 ans. Parmi les jeunes effectivement enquêtés, on compte 823 femmes reparties dans deux groupes d'âges de la manière suivante : 453 femmes sont issues du groupe d'âges 15 - 24 ans et 370 appartiennent au groupe d'âges 25 - 34 ans. Les femmes de ces deux groupes d'âges représentent respectivement $55 \%$ et $45 \%$ de l'ensemble des femmes enquêtées.

Notons que cette enquête a utilisé deux principaux outils de collecte à savoir: un questionnaire ménage classique permettant de collecter des 
données sur les caractéristiques et la composition des ménages au moment de l'enquête et un questionnaire biographique individuel accompagné d'une fiche permettant de retracer la chronologie des événements vécus par les enquêtés appelée fiche AGEVEN (Age-événements). Les méthodes d'analyse utilisées dans cette étude sont essentiellement descriptives. Il s'agit d'explorer l'allure des relations entre les proportions des deux groupes de génération des 15-24 ans et 25-34 ans lors de leur entrée dans la vie génésique et leurs caractéristiques individuelles. Nous utiliserons particulièrement la méthode des tables de séjour de Kaplan-Meier. Ces tables permettent de décrire les événements qui varient au cours du temps en considérant le temps qui s'écoule entre un instant de référence commun à tous les individus soumis au risque de connaître l'événement étudié et la date de l'événement ou de sortie d'observation (Bocquier, 1996). Concrètement, on pourra décrire le calendrier de la survenance de la grossesse ou de la naissance issue des jeunes femmes en fonction du temps et d'autres caractéristiques correspondantes. Des indicateurs résumés comme le temps médian passé dans une période, le premier et éventuellement le troisième quartile sont utilisés pour une description plus précise de la survenance de l'événement.

\section{Resultats et commentaires Âge au premier rapport sexuel}

D'après les résultats du tableau 1, l'intensité de l'entrée en vie sexuelle semble élevée chez les jeunes femmes à Bafia. Chez les 25-34 ans, l'intensité est élevée entre 15 et 21 ans et elle atteint son maximum à 19 ans avec une proportion de 10,2\%. Cependant, les 15-24 ans sont plus actifs entre 15 et 19 ans avec un pic à 16 ans qui correspond à une proportion de $19,9 \%$. On constate que les plus jeunes deviennent de plus en plus précoces en matière d'entrée en vie sexuelle que les plus âgées. En effet, l'âge médian passe de 19 ans pour la cohorte 25-34 ans à 17 ans pour la cohorte 15-24 ans. Les variations selon le niveau de vie montrent que, comparativement aux femmes d'autres catégories socioéconomiques, les femmes de niveau de vie faible entrent de plus en plus massivement $(38,8 \%)$ et tôt dans la vie sexuelle avant l'âge de 17 ans (cf. graphique 1). Les proportions correspondantes pour les femmes de niveaux de vie élevé et moyen sont respectivement de $25,6 \%$ et $34,7 \%$. 
Tableau 1 : Répartition (en \%) des femmes par groupe d'âges selon l'âge au premier rapport sexuel

\begin{tabular}{|c|c|c|c|}
\hline $\begin{array}{l}\text { Âge au premier } \\
\text { rapport sexuel }\end{array}$ & $15-24$ ans & $25-34$ ans & Ensemble \\
\hline 10 & 0,3 & 0,3 & 0,5 \\
\hline 11 & 1,2 & 0,5 & 0,7 \\
\hline 12 & 1,2 & 1,1 & 1,3 \\
\hline 13 & 4,1 & 2,5 & 2,6 \\
\hline 14 & 6,1 & 2,8 & 4,8 \\
\hline 15 & 14,0 & 8,5 & 10,0 \\
\hline 16 & 19,9 & 9,3 & 11,9 \\
\hline 17 & 14,0 & 9,9 & 9,8 \\
\hline 18 & 14,3 & 9,9 & 10,8 \\
\hline 19 & 12,9 & 10,2 & 10,4 \\
\hline 20 & 4,1 & 9,9 & 8,7 \\
\hline 21 & 3,8 & 8,8 & 6,5 \\
\hline 22 & 3,5 & 5,5 & 6,4 \\
\hline 23 & 0,6 & 5,2 & 4,2 \\
\hline 24 & - & 4,4 & 2,7 \\
\hline 25 & - & 5,2 & 3,2 \\
\hline 26 & - & 2,5 & 1,8 \\
\hline 27 & - & 2,2 & 1,8 \\
\hline 28 & - & 0,8 & 1,1 \\
\hline 29 & - & 0,5 & 0,8 \\
\hline Total & 100,0 & 100,0 & 100,0 \\
\hline Âge médian & 17 & 19 & 18 \\
\hline Effectif & 453 & 370 & 823 \\
\hline
\end{tabular}

Graphique 1 : Répartition (en \%) des femmes par tercile de niveau de vie selon leur âge au premier rapport sexuel

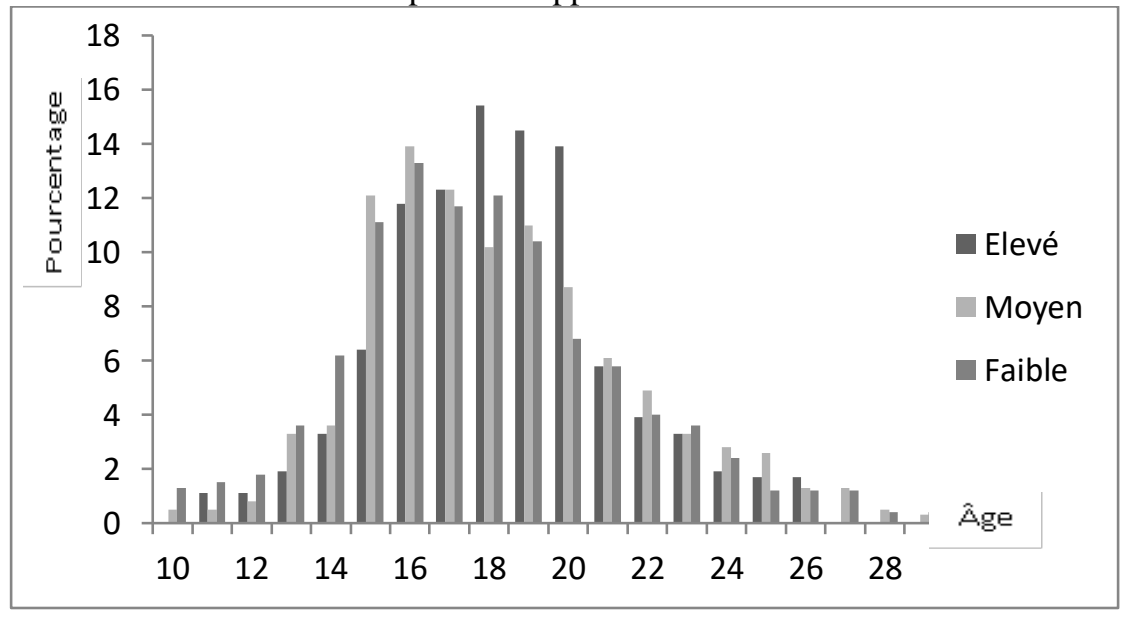




\section{Age au premier mariage}

L'entrée en union est un déterminant de l'entrée dans la vie féconde. L'analyse effectuée dans cette section s'intéresse au calendrier de la primonuptialité des jeunes femmes selon la cohorte et le niveau de vie depuis l'âge de 10 ans. On mesure le temps écoulé entre cet âge et le moment de la première union (ou sortie du célibat) en utilisant les courbes de séjour de Kaplan-Meier. Ainsi, d'après le graphique 2, le mariage n'est pas répandu chez les jeunes femmes à Bafia et celles de 15-24 ans se marient tardivement par rapport à leurs homologues de 25-34 ans. A l'âge de 23 ans, $10 \%$ des 25 34 ans sont déjà entrées en union contre $8 \%$ pour les $15-24$ ans.

Graphique 2 : Proportion des femmes qui sont encore célibataires par cohorte selon leur âge

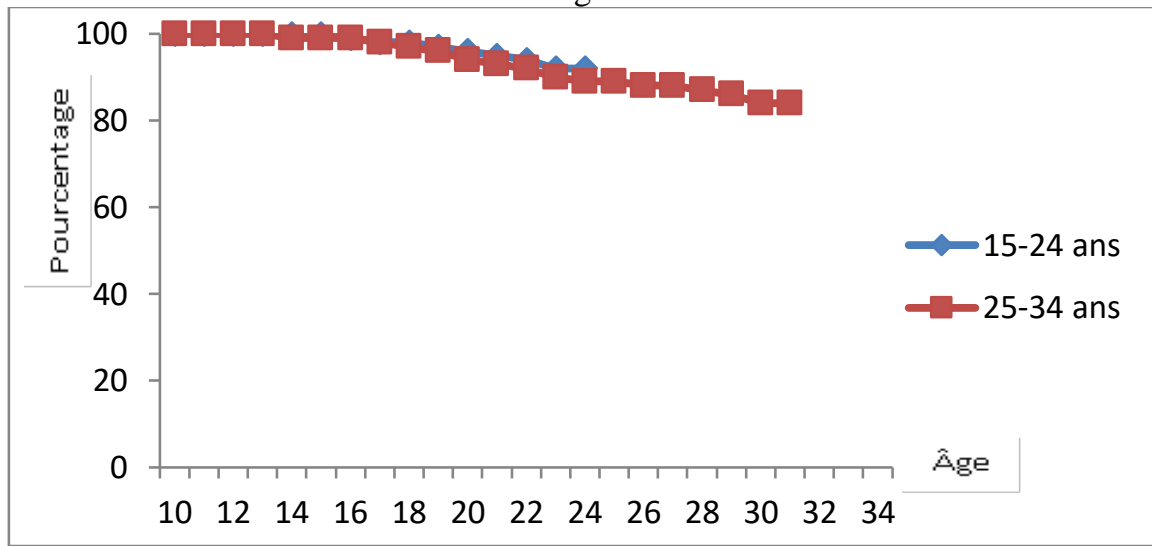

En outre, le niveau de vie a aussi une influence sur le calendrier de la primo-nuptialité. Le graphique 3 montre que les jeunes femmes entrent tardivement en union à mesure que le niveau de vie augmente. Alors que $12 \%$ des femmes de niveau de vie faible sont déjà entrées en union à l'âge de 24 ans, elles ne sont que $8 \%$ pour le niveau de vie élevé.

Graphique 3 : Proportion de jeunes femmes qui ne sont pas encore entrées en union selon leur niveau de vie et leur âge

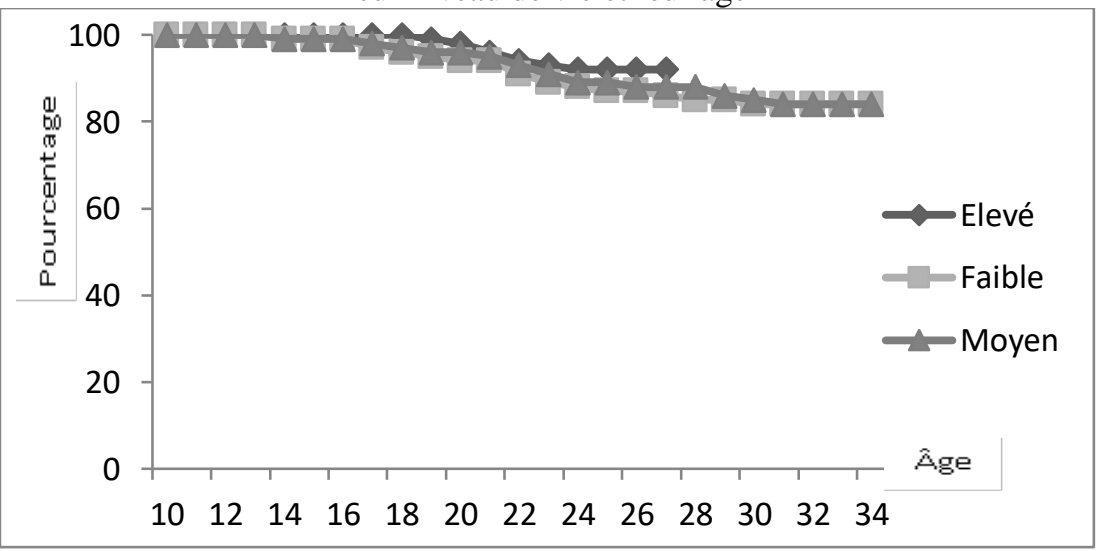




\section{Connaissance et utilisation des methodes contraceptives}

Dans la mesure où l'information conditionne les comportements, la connaissance des méthodes contraceptives est un pré requis pour leur utilisation. Ainsi, pour pouvoir utiliser la contraception, il est indispensable de connaître au moins une méthode contraceptive. Ces différentes méthodes sont classées dans cette étude en deux catégories principales :

- $\quad$ les méthodes modernes qui comprennent la pilule, le stérilet ou DIU (Dispositif Intra Utérin), les injectables, les implants (Norplants), le condom masculin, le condom féminin, la Méthode de l'Allaitement Maternel et l'Aménorrhée (MAMA), le diaphragme, les méthodes vaginales (spermicides, mousses et gelées), la stérilisation féminine et la stérilisation masculine ;

- $\quad$ les méthodes traditionnelles, notamment la continence périodique (ou rythme) et le retrait. Les méthodes dites «populaires », comme les herbes et les tisanes ont été incluses dans cette catégorie.

Il ressort du tableau 2 que le niveau de connaissance des méthodes contraceptives des femmes âgées de 25-34 ans est très élevé que celui des femmes de 15-24 ans quel que soit leur statut matrimonial. En effet, chez les femmes célibataires, $82,8 \%$ des 25-34 ans ont déclaré connaître comment éviter une grossesse contre 74,4\% des 15-24 ans. Il en est de même chez les femmes mariées où les proportions correspondantes sont $72,7 \%$ chez les 25 34 ans et $61,9 \%$ chez les $15-24$ ans.

Quant à la connaissance d'une méthode spécifique, les données du tableau 2 montrent que, quels que soient le groupe d'âges et le statut matrimonial, le condom est la méthode la plus connue. Il est suivi de l'abstinence périodique, de la pilule et des injectables. Ainsi, chez les femmes célibataires âgées de 15 à 24 ans, 91,9\% ont cité le condom, 73,1\% l'abstinence périodique, $51,3 \%$ la pilule, et $29,4 \%$ les injectables. Les proportions des femmes célibataires du groupe d'âges 25-34 ans correspondantes sont $89,6 \% ; 65,3 \% ; 55,4 \%$ et $33,3 \%$. On observe les mêmes tendances chez les femmes mariées avec des proportions respectivement de $84,6 \% ; 69,2 \% ; 38,5 \%$ et $23,1 \%$ chez les $15-24$ ans et de $82,1 \% ; 69,6 \% ; 48,2 \%$ et $33,9 \%$ chez les $25-34$ ans. Par ailleurs, la majorité des femmes (plus de 78\%) ont une connaissance des méthodes contraceptives modernes, quels que soient leur groupe d'âges et leur statut matrimonial.

Tableau 2 : Répartition (en \%) des femmes qui connaissent une méthode contraceptive avant de tomber enceinte par groupe d'âges et par statut matrimonial

\begin{tabular}{|c|c|c|c|c|c|}
\hline $\begin{array}{c}\text { Connaissance des méthodes } \\
\text { contraceptives }\end{array}$ & \multicolumn{2}{|c|}{$\begin{array}{c}\text { Femmes } \\
\text { célibataires }\end{array}$} & \multicolumn{2}{|c|}{ Femmes mariées } & \multirow{2}{*}{ Ensemble } \\
\cline { 2 - 5 } & $15-24$ & $25-34$ & $15-24$ & $25-34$ & \\
\hline $\begin{array}{c}\text { Connaissez-vous des méthodes } \\
\text { permettant d'éviter une grossesse ? }\end{array}$ & & & & & \\
\hline
\end{tabular}




\begin{tabular}{|c|c|c|c|c|c|}
\hline Oui & 74,4 & 82,8 & 61,9 & 72,7 & 77,6 \\
Non & 25,6 & 17,2 & 38,1 & 27,3 & 22,4 \\
\hline Total & 100,0 & 100,0 & 100,0 & 100,0 & 100,0 \\
\hline Si oui, quelle(s) méthode(s) ? & & & & & \\
Abstinence périodique & 73,1 & 65,3 & 69,2 & 69,6 & 68,7 \\
Condom & 91,9 & 89,6 & 84,6 & 82,1 & 89,4 \\
Pilule & 51,3 & 55,4 & 38,5 & 48,2 & 52,6 \\
DIU & 11,3 & 22,5 & 0,0 & 21,4 & 17,7 \\
Stérilet & 11,9 & 10,8 & 0,0 & 12,5 & 11,1 \\
Injectables & 29,4 & 33,3 & 23,1 & 33,9 & 31,7 \\
Implants & 16,9 & 17,1 & 23,1 & 17,9 & 17,3 \\
Retrait & 20,0 & 18,5 & 0,0 & 17,9 & 18,4 \\
\hline Type de méthode & & & & & \\
Méthode moderne & 78,7 & 80,2 & 100,0 & 80,3 & 80,3 \\
Méthode traditionnelle & 21,3 & 19,8 & 0,0 & 19,7 & 19,7 \\
\hline Total & 100,0 & 100,0 & 100,0 & 100,0 & 100,0 \\
\hline Effectif & 428 & 293 & 25 & 77 & 823 \\
\hline
\end{tabular}

Le tableau 3 donne la répartition des femmes ayant une connaissance des méthodes de contraception selon certaines caractéristiques sociodémographiques et économiques. Il apparaît que les proportions des femmes connaissant les méthodes contraceptives, et en particulier les méthodes modernes augmentent en fonction de leur niveau de vie et de leur niveau d'instruction quels que soient le groupe d'âges et le statut matrimonial. En effet, les femmes célibataires vivant dans les ménages pauvres $(76,3 \%$ pour les $15-24$ ans et $70 \%$ pour les $25-34$ ans) et sans instruction (60\% pour les $15-24$ ans et $66,7 \%$ pour les $25-34$ ans) sont proportionnellement moins nombreuses à connaître les méthodes de contraception modernes que leurs homologues des ménages riches $(85,7 \%$ pour les $15-24$ ans et $82,2 \%$ pour les $25-34$ ans) et de niveau d'instruction primaire (78,5\% pour les $15-24$ ans et $80,4 \%$ pour les $25-34$ ans). De même, chez les femmes mariées âgées de 25-34 ans, les proportions des personnes pauvres et non instruites connaissant ce type de méthode sont respectivement $60 \%$ et $50 \%$ contre $84,6 \%$ et $78,6 \%$ des personnes respectivement riches et de niveau d'instruction primaire.

Tableau 3: Répartition (en \%) des femmes ayant connaissance des méthodes contraceptives par groupe d'âges et par statut matrimonial selon le niveau de vie du ménage et le niveau d'instruction

\begin{tabular}{|c|c|c|c|c|}
\hline \multirow{2}{*}{ Type de méthodes } & \multicolumn{2}{c|}{ Femmes célibataires } & \multicolumn{2}{c|}{ Femmes mariées } \\
\cline { 2 - 5 } & $15-24$ & $25-34$ & $15-24$ & $25-34$ \\
\hline \multicolumn{4}{|c|}{ Niveau de vie du ménage } \\
\hline Méthodes modernes & 76,3 & 70,0 & 100,0 & 60,0 \\
Pauvre & 79,8 & 80,6 & 100,0 & 75,0 \\
Moyen &
\end{tabular}




\begin{tabular}{|c|c|c|c|c|}
\hline Riche & 85,7 & 82,2 & 100,0 & 84,6 \\
\hline Méthodes traditionnelles & 23,7 & 30,0 & 0,0 & 40,0 \\
Pauvre & 20,2 & 19,4 & 0,0 & 25,0 \\
Moyen & 14,3 & 17,8 & 0,0 & 15,4 \\
Riche & Instruction \\
\hline \multicolumn{5}{|l}{} \\
Méthodes modernes & 60,0 & 66,7 & 0,0 & 50,0 \\
Aucun niveau & 78,5 & 80,4 & 100,0 & 78,6 \\
Primaire & 84,0 & 81,5 & 100,0 & 82,5 \\
Secondaire et plus & 40,0 & 33,3 & 0,0 & 50,0 \\
Méthodes traditionnelles & 21,5 & 19,6 & 0,0 & 21,4 \\
Aucun niveau & 16,0 & 18,5 & 0,0 & 17,5 \\
Primaire & 428 & 293 & 25 & 77 \\
\hline Secondaire et plus &
\end{tabular}

Les jeunes femmes ayant contracté au moins une grossesse ont été interrogées sur la principale méthode contraceptive qu'elles utilisaient avant de tomber enceinte. Le tableau 4 suivant présente leurs réponses par rapport à la première et à la dernière grossesse qu'elles ont eues. Il en ressort que, plus de la moitié des femmes célibataires, soient $62,7 \%$ des $15-24$ ans et 54,6\% des 25-34 ans, utilisaient une méthode contraceptive avant de tomber enceinte pour la première fois. Par contre, moins de la moitié des femmes mariées (46,2 \% des 15-24 ans et 41,7\% des 25-34 ans) se trouvent dans cette situation. La méthode la plus utilisée était le condom chez les femmes célibataires (72,3\% pour les $15-24$ ans et $59,5 \%$ pour les $25-34$ ans) et l'abstinence périodique chez les femmes mariées (50\% pour les 15-24 ans et $40 \%$ pour les $25-34$ ans).

On observe les mêmes tendances pour l'utilisation de la contraception avant la dernière grossesse. Les proportions des femmes qui utilisent les méthodes contraceptives avant leur dernière grossesse sont plus élevées chez les femmes célibataires $(62,5 \%$ des $15-24$ ans et 54,9\% des 25 34 ans) que chez les femmes mariées $(38,5 \%$ des $15-24$ ans et $46,4 \%$ des 25-34 ans). La méthode la plus utilisée reste le condom chez les femmes célibataires (72\% pour les $15-24$ ans et $62,3 \%$ pour les $25-34$ ans) et l'abstinence périodique chez les femmes mariées (60\% pour les 15-24 ans et $34,6 \%$ pour les 25-34 ans). Enfin, signalons que les femmes mariées âgées de 25 à 34 ans ont fait recours aussi au condom dans la même proportion que celle de l'abstinence périodique avant leur dernière grossesse.

Tableau 4 : Répartition (en \%) des femmes par groupe d'âges et par statut matrimonial selon l'utilisation de méthodes contraceptives

\begin{tabular}{|c|c|c|c|c|c|}
\hline Recours aux méthodes & \multicolumn{2}{|c|}{ Femmes célibataires } & \multicolumn{2}{|c|}{ Femmes mariées } & Ensemble \\
contraceptives & $15-24$ & $25-34$ & $15-24$ & $25-34$ & \\
\hline
\end{tabular}




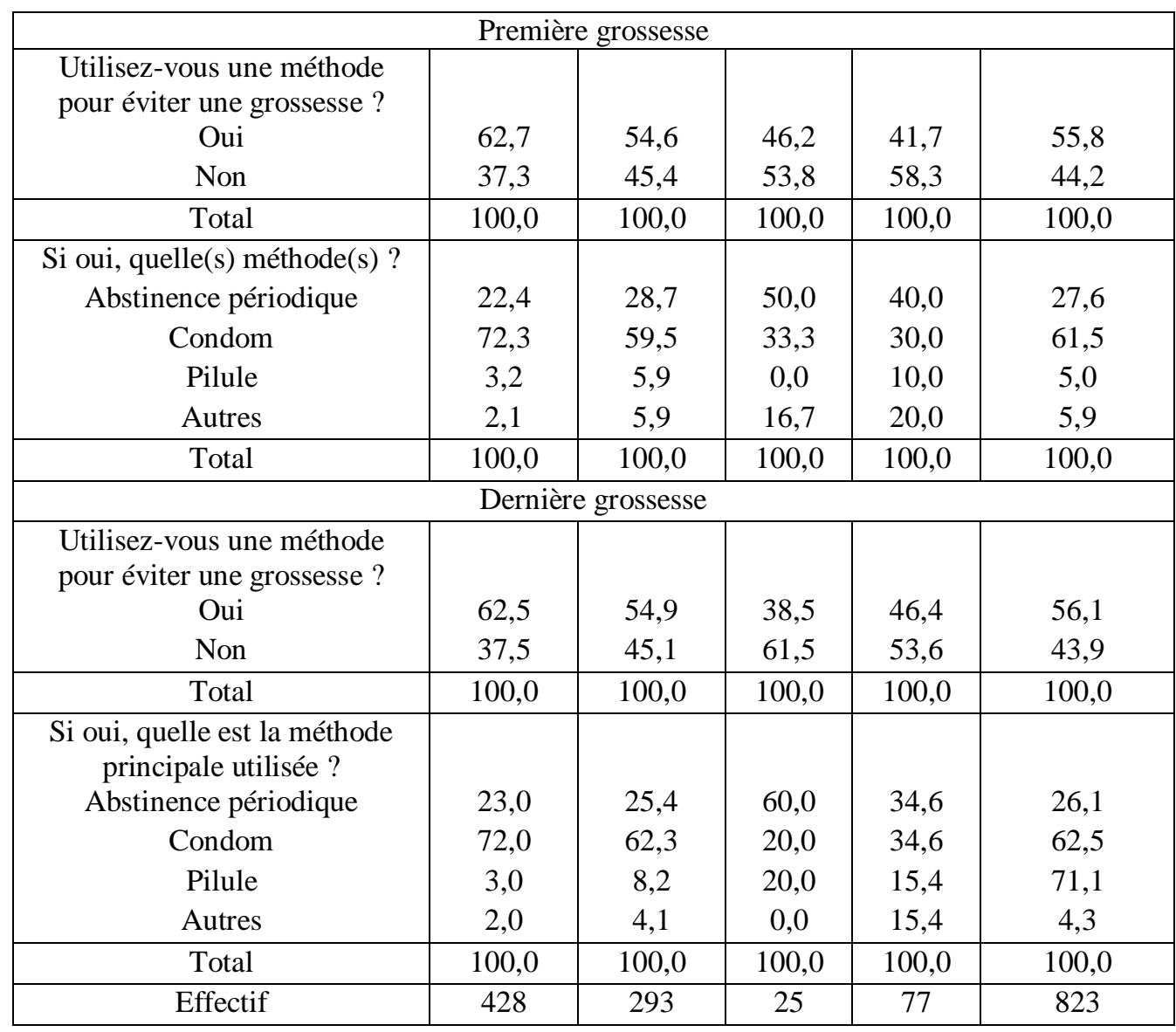

\section{Entree dans la vie genesique}

Parmi les 823 jeunes femmes enquêtées, on dénombre 242 (29,4\%) qui n'ont jamais été enceintes, 199 n'en sont qu'à leur première grossesse $(24,2 \%)$. Les grossesses de rang supérieur représentent respectivement $17,9 \%$ pour les grossesses de rang $2,11,9 \%$ pour le rang $3,9,4 \%$ pour le rang $4,5 \%$ pour le rang 5 et moins de $1,5 \%$ pour les rangs 6 ou plus.

D'après le tableau 5, l'âge moyen à la première grossesse est précoce chez les 15-24 ans que chez les 25-34 ans, soient respectivement 17,7 ans contre 20,1 ans pour les célibataires, et 17,6 ans contre 19,4 ans pour les mariées. Comme on pouvait s'y attendre, le nombre de grossesse est plus élevé chez les 25-34 ans que chez les 15-24 ans quel soit leur statut matrimonial. Les célibataires du groupe d'âges 25-34 ans ont contracté en moyenne 2,6 grossesses contre 0,8 pour leurs homologues du groupe 15-24 ans. Les moyennes pour les mariées sont respectivement 3,6 pour les 25-34 ans et 1,6 pour les $15-24$ ans. 
Tableau 5: Indicateurs de l'histoire génésique des femmes par groupe d'âges et par statut matrimonial

\begin{tabular}{|c|c|c|c|c|c|}
\hline \multirow{2}{*}{} & \multicolumn{2}{|c|}{$\begin{array}{c}\text { Femmes } \\
\text { célibataires }\end{array}$} & \multicolumn{2}{c|}{ Femmes mariées } & \multirow{2}{*}{ Ensemble } \\
\cline { 2 - 5 } & $15-24$ & $25-34$ & $15-24$ & $25-34$ & \\
\hline A déjà eu une grossesse & & & & & \\
Oui & 50,2 & 91,8 & 84,0 & 98,7 & 70,6 \\
Non & 49,8 & 8,2 & 16,0 & 1,3 & 29,4 \\
\hline Total & 100,0 & 100,0 & 100,0 & 100,0 & 100,0 \\
\hline Age moyen à la première & 17,7 & 20,1 & 17,6 & 19,4 & 19,0 \\
grossesse & & & & & \\
\hline Nombre moyen de grossesse & 0,8 & 2,6 & 1,6 & 3,6 & 1,7 \\
Médiane & 1 & 2 & 2 & 3 & 1 \\
Minimum & 0 & 0 & 0 & 0 & 0 \\
Maximum & 5 & 8 & 4 & 8 & 8 \\
\hline Effectif & 428 & 293 & 25 & 77 & 823 \\
\hline
\end{tabular}

Le tableau 6 présente la répartition des femmes de 15-34 ans selon leur âge à la survenance de la première grossesse et leur statut matrimonial. On en déduit que la proportion des femmes ayant eu au moins une grossesse augmente très rapidement avec l'âge après l'âge de 15 ans et atteint son maximum à l'âge de 18 ans puis elle baisse progressivement quel que soit le statut matrimonial. Ainsi, $10,1 \%$ et $11,4 \%$ des femmes célibataires et mariées respectivement ont déjà eu au moins une grossesse à l'âge de 16 ans. Ces proportions atteignent, à l'âge de 18 ans, $15,7 \%$ chez les célibataires et $18,6 \%$ chez les mariées.

Tableau 6 : Répartition (en \%) des femmes par âge à la survenance de la première grossesse selon leur statut matrimonial

\begin{tabular}{|c|c|c|}
\hline \multirow{2}{*}{$\begin{array}{c}\text { Age à la survenance de la } \\
\text { première grossesse }\end{array}$} & \multicolumn{2}{|c|}{ Statut matrimonial à la première grossesse } \\
\cline { 2 - 3 } 12 & Célibataire & Mariée \\
\hline 13 & 0,2 & 1,0 \\
14 & 3,3 & 1,0 \\
15 & 4,4 & 1,0 \\
16 & 4,8 & 6,2 \\
17 & 10,1 & 11,4 \\
18 & 11,6 & 13,3 \\
19 & 15,7 & 18,6 \\
20 & 14,5 & 11,3 \\
21 & 8,5 & 8,4 \\
22 & 6,4 & 6,2 \\
23 & 5,2 & 6,2 \\
24 & 4,6 & 4,1 \\
25 & 3,3 & 4,1 \\
26 & 2,7 & 3,1 \\
\hline
\end{tabular}




\begin{tabular}{|c|c|c|}
\hline 27 & 0,6 & 1,0 \\
$28 \mathrm{ou}+$ & 2,7 & 1,0 \\
\hline Total & 100,0 & 100,0 \\
\hline
\end{tabular}

Le tableau 7 met en exergue la proportion des femmes ayant eu une grossesse par groupe d'âges et par statut matrimonial selon le niveau de vie du ménage et leur niveau d'instruction. En effet, la survenance d'une grossesse peut être influencée par le niveau de vie du ménage de la femme et son éducation. D'une part, l'appartenance à un ménage aisé par exemple, pourrait inciter les jeunes femmes à contracter une grossesse compte tenu des conditions de vie meilleures; et d'autre part, l'instruction pourrait constituer un frein à la grossesse. D'après ce tableau, le niveau de vie du ménage agit comme s'il y a un effet de seuil. En effet, quel que soit le statut matrimonial, les proportions des femmes ayant contracté une grossesse sont plus élevées dans les ménages de niveau de vie moyen que dans les autres types de ménages. Par contre, c'est dans les ménages riches que ces proportions sont les plus faibles. A titre d'illustration, près de la moitié des femmes célibataires âgées de 15 à 24 ans et vivant dans les ménages de niveau de vie moyen ont contracté une grossesse contre $37,2 \%$ et $13,7 \%$ de leurs congénères vivant dans les ménages pauvres et riches respectivement.

Par ailleurs, on constate que l'instruction a une influence négative chez les 15-24 ans quel que soit leur statut matrimonial. En effet, trois quarts des femmes célibataires âgées de 15 à 24 ans et ayant un niveau d'instruction primaire ou moins ont contracté une grossesse contre seulement un quart de leurs homologues du niveau secondaire ou plus. Les proportions correspondantes pour les femmes mariées du même groupe d'âges sont $62,4 \%$ (pour le niveau primaire ou moins) et $37,6 \%$ (pour le niveau secondaire ou plus).

En outre, la plupart des grossesses contractées par les femmes mariées ont une meilleure issue que celles des célibataires quel que soit leur groupe d'âges. En effet, 87,7\% de grossesses chez les femmes mariées âgées de 25 à 34 ans ont abouti à une naissance vivante contre 79,7\% chez leurs consœurs célibataires du même groupe d'âges. De même, les proportions chez les mariées et chez les célibataires âgées de 15 à 24 ans sont respectivement $76,8 \%$ et $74 \%$. Enfin, les cas d'avortements spontanés ou provoqués sont relativement plus nombreux chez les célibataires que chez les mariées.

Tableau 7 : Répartition (en \%) des femmes ayant eu une grossesse par groupe d'âges et par statut matrimonial selon le niveau de vie du ménage et leur niveau d'instruction

\begin{tabular}{|c|c|c|c|c|}
\hline \multirow{2}{*}{ Caractéristiques } & \multicolumn{2}{|c|}{ Femmes célibataires } & \multicolumn{2}{c|}{ Femmes mariées } \\
\cline { 2 - 5 } & $15-24$ & $25-34$ & $15-24$ & $25-34$ \\
\hline $\begin{array}{c}\text { Niveau de vie } \\
\text { Pauvre }\end{array}$ & 37,2 & 34,9 & 37,6 & 30,6 \\
\hline
\end{tabular}




\begin{tabular}{|c|c|c|c|c|}
\hline Moyen & 49,1 & 46,9 & 47,6 & 51,5 \\
Riche & 13,7 & 18,2 & 14,8 & 17,9 \\
\hline Niveau d'instruction & & & & \\
Primaire ou moins & 74,9 & 45,3 & 62,4 & 43,4 \\
Secondaire ou plus & 25,1 & 54,7 & 37,6 & 56,6 \\
\hline Total & 100,0 & 100,0 & 100,0 & 100,0 \\
\hline Issue des grossesses (nombre total) & & & & \\
Grossesse en cours & 8,8 & 2,7 & 12,2 & 1,1 \\
Naissances vivantes & 74,0 & 79,7 & 76,8 & 87,7 \\
Morts nés & 2,1 & 2,5 & 2,4 & 3,6 \\
Avortements spontanés & 8,0 & 7,4 & 3,7 & 5,4 \\
Avortements provoqués & 7,1 & 7,7 & 4,9 & 2,2 \\
\hline Total & 100,0 & 100,0 & 100,0 & 100,0 \\
\hline
\end{tabular}

\section{Conclusion}

Cette étude a eu pour objectif principal de faire une analyse différentielle des comportements de fécondité des jeunes femmes à Bafia, à partir des données de l'enquête biographique intitulée pauvreté et besoins non satisfaits en santé de la reproduction des adolescents et des jeunes résidant dans cette ville en 2010. Une telle analyse est susceptible d'aider à identifier certains groupes cibles d'une part, et permettre aux décideurs d'orienter leurs actions en matière de la santé reproductive des jeunes d'autre part.

Les comportements de fécondité ont été appréhendés à travers l'âge au premier rapport sexuel et au premier mariage, le niveau de connaissances des jeunes femmes sur les méthodes contraceptives et l'utilisation de ces méthodes, leur âge à la première grossesse et à l'accouchement. L'approche utilisée mesure l'intensité du phénomène à partir de ces indicateurs et les met en relation avec les caractéristiques sociodémographiques, culturelles et économiques des femmes. Les résultats de l'analyse révèlent des écarts importants en matière de comportements de fécondité entre les femmes du groupe d'âges 15-24 ans et celles de 25-34 ans selon leurs caractéristiques.

Les femmes de 15-24 ans deviennent de plus en plus précoces en matière d'entrée en vie sexuelle que celles de 25-34 ans. De même, l'âge moyen à la première grossesse est précoce chez les 15-24 ans que chez les 25-34 ans, soient respectivement 17,7 ans contre 20,1 ans pour les célibataires, et 17,6 ans contre 19,4 ans pour les mariées. En revanche, les jeunes femmes de 15-24 ans se marient tardivement par rapport à leurs homologues de 25-34 ans. Par ailleurs, plus de la moitié des femmes célibataires, soient $62,7 \%$ des 15-24 ans et 54,6\% des 25-34 ans, utilisaient une méthode contraceptive avant de tomber enceinte pour la première fois. Par contre, moins de la moitié des femmes mariées (46,2 \% des 15-24 ans et 
41,7 \% des 25-34 ans) se trouvent dans cette situation. Le niveau de vie du ménage et l'instruction ont une influence sur ces indicateurs. Ces résultats devraient contribuer à une meilleure connaissance des comportements reproductifs des jeunes femmes au Cameroun en général, et à Bafia en particulier.

\section{References:}

1. Beninguissé Gervais (2007), «Sexualité pré maritale et santé de la reproduction des adolescents et des jeunes en Afrique subsaharienne ", L'Afrique face à ses défis démographiques : Un avenir incertain, Paris; Nogent-sur-Marne, pp. 289-321.

2. Bessala Pascal Yannick (2015), Dépenses des ménages en éducation au Cameroun: essai d'explication des inégalités dans l'arrondissement de Bafia. Mémoire de fin de formation, Institut de Formation et de Recherche Démographiques (IFORD), université de Yaoundé II, 157p.

3. Bocquier Philippe (1996), L'analyse des enquêtes biographiques à l'aide du logiciel STATA, Documents et manuels du CEPED $\mathrm{n}^{\circ} 4$, $208 p$.

4. Bureau Central de Recensement et d'Etudes Démographiques (BUCREP), 2011. Rapport final de l'Enquête Démographique et de Santé et à Indicateurs Multiples du Cameroun, Yaoundé.

5. Davis K. et Blake J. (1956). Social structure and fertility : An analytic framework, Economic development and cultural change, $\mathrm{n}^{\circ} 4$, p. 211 .

6. Guillaume A., Khlat M. (2004), Santé de la reproduction au temps du Sida en Afrique, Les Collections du CePeD, 154p.

7. Institut National de la Statistique du Cameroun (INS), 2008, Conditions de vie des populations et profil de pauvreté au Cameroun en 2007. Chapitre 4: situation a Bafia, Août 2008,143 p. OMS (1999). Santé et Développement de l'adolescent, pour une programmation efficace, Rapport d'un groupe d'étude OMS, FNUAP, UNICEF sur la programmation relative à la santé des adolescents, $306 \mathrm{p}$.

8. Schoumaker B. et Tabutin D. (1999). Relation entre pauvreté et fécondité dans les pays du Sud : Connaissances, méthodologie et illustrations, Document de travail $n^{\circ} 2$, Université Catholique de Louvain, SPED.

9. Vimard P. (2007). Changements contemporains en fécondité et santé de la reproduction dans les pays du Sud, in, Adjamagbo A., Mselatti P. et Vimard P. (éds), Santé de la reproduction et fécondité dans les 
pays du Sud. Nouveaux contextes et nouveaux comportements, Louvain-la-Neuve, Academia-Bruylant, pp.11-35.

10. Wang Sonnè, (1998), «Approche historique des quinze premières années de la lutte contre la maladie du sommeil à Bafia et dans le Mbam 1921-1935 », in Histoire en Afrique Centrale, Institut de Recherche pour le Développement, Paris. 